\title{
A cross-sectional survey of methods for controling hand-foot syndrome in patients receiving capecitabine treatment
}

\author{
SHINYA SUZUKI ${ }^{1}$, SHUICHI NAWATA ${ }^{2}$, YUSUKE INADA ${ }^{3}$, DAISUKE SATO ${ }^{4}$, JUNICHI KUSANO ${ }^{1}$, \\ DAISUKE ICHIKURA ${ }^{2}$, KAZUHIRO TORIGOE $^{5}$, KAZUMI ISHITSUKA $^{5}$, FUMIAKI SATO $^{6}$, \\ HIROYASU SAKAI $^{6}$ and TETSURO YUMOTO ${ }^{6}$
}

\author{
${ }^{1}$ Department of Pharmacy, Kanagawa Prefectural Keiyukai Keiyu Hospital, Yokohama, Kanagawa 220-8521; \\ ${ }^{2}$ Department of Hospital Pharmaceutics, Showa University Northern Yokohama Hospital, Yokohama, Kanagawa 224-8503; \\ ${ }^{3}$ Department of Pharmaceutical Services, Yokohama Rosai Hospital, Yokohama, Kanagawa 222-0036; ${ }^{4}$ Department of \\ Pharmacy, Fujisawa City Hospital, Fujisawa, Kanagawa 251-8550; ${ }^{5}$ Division of Applied Pharmaceutical Education \\ and Research, and ${ }^{6}$ Department of Analytical Pathophysiology, Hoshi University, Tokyo 142-8501, Japan
}

Received February 19, 2018; Accepted August 1, 2018

DOI: $10.3892 / \mathrm{mco} .2018 .1689$

\begin{abstract}
Medical personnel actively provide patients taking capecitabine with information on the items to prevent and treat hand-foot syndrome (HFS). However, they are typically unable to ascertain the extent of patient compliance with the recommended items. Thus, the aim of the present study was to ascertain the association between patient compliance with preventative measures for HFS and the development of HFS. Subjects included 90 patients who were treated with a drug regimen that included capecitabine. Patients were treated at one of four facilities between July 2015 and January 2017. The main parameters studied were the extent to which items to prevent and treat HFS were (or were not) followed, and the associaiton between this extent and the development of HFS symptoms. A manual prepared by a pharmaceutical company that manufactures capecitabine describes 15 routine items to follow in order to prevent and treat HFS. The two activities patients most often performed were 'applying a moisturizer' (74.1\%) and 'keeping one's skin clean (e.g., washing one's hands and feet)' (64.7\%). The two activities patients least often performed were 'using sunscreen on exposed areas' (14.1\%) and 'using soft insoles' (11.8\%). Patients who performed more items to prevent and treat HFS were significantly less likely to develop symptoms of HFS ( $\mathrm{P}=0.022)$. Based on these findings, it is recommended that medical personnel provide instructions to the patients regarding the specific items necessary to prevent and treat HFS, and to follow-up with the patients regarding
\end{abstract}

Correspondence to: Dr Tetsuro Yumoto, Department of Analytical Pathophysiology, Hoshi University, 2-4-41 Ebara, Shinagawa, Tokyo 142-8501, Japan

E-mail: t-yumoto@hoshi.ac.jp

Key words: hand-foot syndrome, capecitabine, patient management, moisturizer, hand-foot syndrome atlas their compliance, with an emphasis on the items they are less likely to take and on the instructions to avoid external irritants. Following these guidelines should lead to qualitative improvement in HFS management.

\section{Introduction}

Capecitabine is a fluorinated pyrimidine anticancer drug demonstrated to be non-inferior to 5-fluorouracil/leucovorin treatment in the X-ACT study (1). Capecitabine is also considered a standard postoperative adjuvant chemotherapy for colorectal cancer, according to the Colorectal Cancer Treatment Guidelines (2). However, hand-foot syndrome (HFS) is a frequent adverse reaction to capecitabine, and the underlying mechanisms of the onset of HFS are unclear. Histologically, the inhibition of the proliferation of epidermal basal cells and drug secretion from exocrine sweat glands are suspected mechanisms of the onset of HFS (3). Major symptoms of HFS are erythema, dysesthesia, pain, skin cracks, and desquamation of the palms and soles of the feet (4). The clinical manifestations of HFS are reversible and non-life-threatening, but they often markedly affect a patient's quality of life. Because these manifestations necessitate a modification or discontinuation of treatment, a greater understanding of their prevention and treatment is necessary. Currently, there are no established techniques to prevent or treat HFS, and research has pointed to the benefits of symptomatic treatment with urea ointments, moisturizers, topical steroids, or by wearing gloves at night to retain moisture (5-9). In light of these recommendations, medical personnel typically use brochures or manuals prepared by pharmaceutical companies to actively inform patients taking capecitabine of the various items they can take to prevent and treat HFS. These efforts and approaches have been described at relevant academic conferences $(10,11)$. However, medical personnel cannot ascertain the extent to which patients have (or have not) taken the items to prevent and treat HFS. Thus, we hypothesized that ascertaining the extent to which patients have (or have not) taken these items 
in a cross-sectional study may lead to more efficient patient interventions. Improved forms of intervention by medical personnel based on the current results should reduce the incidence of HFS through the qualitative improvement of patient management, and improve the effectiveness of treatment in the event that HFS develops.

Accordingly, the aim of the current study was to ascertain the relationship between the extent to which patients have taken (or not taken) the items to prevent and treat HFS and the development of HFS.

\section{Subjects and methods}

Subjects. Subjects consisted of 90 patients who underwent at least one course of treatment with a drug regimen that included capecitabine. Patients were treated at one of four facilities (Kanagawa Prefectural Keiyukai Keiyu Hospital, Showa University Northern Yokohama Hospital, Yokohama Rosai Hospital or Fujisawa City Hospital) from July 2015 to January 2017.

Potential subjects were given a detailed explanation of the study. The included patients voluntarily consented to participate in this study and were $\geq 20$ years of age when they provided consent. Individuals were excluded if they were deemed unable to provide informed consent or ineligible for other reasons.

Methodology and items studied. The present study was conducted only one time per patient using patient survey and pharmacist survey forms. The main items studied were the extent to which items to prevent and treat HFS were (or were not) taken, the relationship between the number of items taken to prevent and treat HFS and the development of symptoms of HFS, and the differences between the patients' subjective symptoms of HFS and those assessed by a pharmacist using the Common Terminology Criteria for Adverse Events (CTCAE) Version 4.0. If HFS was grade 0 , the patient was deemed to not have developed HFS; if HFS was grade 1 or 2, the patient was deemed to have developed HFS. The items that were taken for preventing and treating HFS were routine items listed in the HFS Atlas (Chugai Pharmaceutical Co. Ltd, Tokyo, Japan) (12), which is a brochure prepared by a pharmaceutical company. This brochure is widely disseminated domestically, and it is cited in the HFS volume of the Manuals for Management of Serious Adverse Drug Reactions issued by the Ministry of Health, Labour and Welfare of Japan. There are no quantitative criteria for each routine item; hence, the extent to which an item has been taken is subjectively rated by the patient.

Statistical analysis and ethical considerations. The responses to the individual survey items were simply tallied, and the factors affecting the presence or absence of HFS symptoms were examined using univariate analysis (significance level: $5 \%$ ). In order to examine the relationship between the extent to which items to prevent and treat HFS were (or were not) taken and the development of symptoms of HFS, the Wilcoxon rank-sum test was performed with 'the presence or absence of symptoms of HFS as determined by a pharmacist' serving as the dependent variable and 'the number of items (out of a 15 total) in the HFS Atlas (12) that were (or were

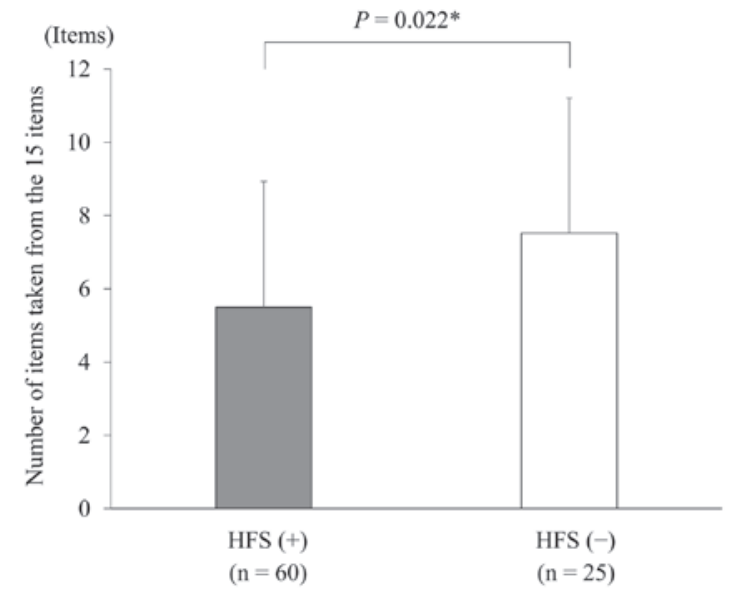

Figure 1. Association between the number of items taken to prevent and treat HFS, and the development of symptoms of HFS. Data shown are the mean \pm standard deviation. ${ }^{*} \mathrm{P}<0.05$, Wilcoxon rank-sum test. HFS, hand-foot syndrome.

not) taken to prevent and treat HFS' serving as the explanatory variable. To examine the relationship between the individual items taken to prevent and treat HFS and the development of symptoms of HFS, Pearson's chi-squared test or Fisher's exact test was performed with 'the presence or absence of symptoms of HFS as determined by a pharmacist' serving as the dependent variable and 'the number of individual items taken (out of a 15 total in the HFS Atlas)' (12) serving as the explanatory variable. In order to examine the differences between a patient's subjective symptoms of HFS and the assessment of HFS by a pharmacist, Fisher's exact test was performed with 'the presence or absence of symptoms of HFS as determined by a pharmacist' serving as the dependent variable and 'the patient's symptoms of HFS' serving as the explanatory variable.

The statistical software JMP ${ }^{\circledR} 12.2$ (SAS Institute, Cary, NC, USA) was used for analysis. This study was ethically reviewed and approved by the Ethics Committee of Keiyu Hospital (Yokohama, Japan), Showa University Northern Yokohama Hospital (Yokohama, Japan), Yokohama Rosai Hospital (Yokohama, Japan), Fujisawa City Hospital (Fujisawa, Japan), and Hoshi University (Tokyo, Japan).

\section{Results}

Patient characteristics. Subjects consisted of 90 patients; 54 males and 36 females. Patients had a median age of 66 years, and the median cumulative dose of capecitabine administered was $105,000 \mathrm{mg}$. The patient characteristics are shown in Table I.

Extent to which items to prevent and treat HFS were (or were not) taken. Of the 90 patients, $78(88.9 \%)$ were prophylactically prescribed an ointment for HFS. All 78 patients were prescribed an ointment containing heparin or an analogue. Two of the 78 patients also used an ointment containing urea. Of the 90 patients, 49 were prescribed vitamin B6. The extent to which the 15 items in the HFS Atlas (12) were (or were not) followed is shown in Table II. Eighty-five of the 90 patients had 
followed at least some of the 15 items in the HFS Atlas (12). The items those 85 patients most often took were 'applying a moisturizer' (74.1\%), 'keeping one's skin clean (e.g., washing one's hands and feet)' (64.7\%), and 'avoiding strenuous exercise (e.g., long walks, jogging, or aerobics)' (55.3\%). The items that the 85 patients least often took were 'using sunscreen on exposed areas' (14.1\%) and 'using soft insoles' (11.8\%).

Association between the number and extent of items taken to prevent and treat HFS and the development of HFS symptoms. The relationship between the number of items taken to prevent and treat HFS and the development of HFS symptoms was also examined. Our results indicated that patients who developed symptoms of HFS had taken fewer items to prevent and treat HFS, while patients who did not develop symptoms of HFS had taken more items to prevent and treat HFS ( $\mathrm{P}=0.022)$ (Fig. 1). The relationship between the extent to which individual items in the HFS Atlas (12) were (or were not) taken and the development of symptoms of HFS was also examined. Our results indicated that patients differed significantly in terms of three items: 'avoiding activities that place pressure on the fingers or palms (e.g., acupressure and writing for long periods)' [odds ratio $(\mathrm{OR})=0.36 ; 95 \%$ confidence interval $(\mathrm{CI})=0.14-0.96 ; \mathrm{P}=0.038$ ], 'not wearing tight-fitting footwear or socks' $(\mathrm{OR}=0.29 ; 95 \% \mathrm{CI}=0.11-0.79 ; \mathrm{P}=0.013)$, and 'refraining from taking hot baths or showers' $(\mathrm{OR}=0.35$; 95\% $\mathrm{CI}=0.13-0.92 ; \mathrm{P}=0.031$ ). Patients who took these three items had a significantly lower incidence of symptoms of HFS (Table II).

Differences in a patient's subjective symptoms of HFS and a pharmacist's assessment of HFS. The differences in the patient's subjective symptoms of HFS and a pharmacist's assessment of HFS were examined. Results indicated significant differences in 'the presence or absence of symptoms of HFS as determined by a pharmacist' and 'the patient's subjective symptoms of HFS' $(\mathrm{P}=0.0002)$. In $18.9 \%$ of patients, the patient reported symptoms related to HFS, but the pharmacist determined that the patient had no symptoms of HFS (Table III).

\section{Discussion}

The current study surveyed patients who were being treated with a drug regimen that included capecitabine, and it ascertained the extent to which items to prevent and treat HFS were (or were not) taken. Results of the present study reveal that patients who took more items for preventing and treating HFS (as listed in the HFS Atlas) (12) had a lower incidence of symptoms of HFS than patients who did not take those items. There are no guaranteed items to prevent and treat HFS, and definitive treatment of HFS is achieved via a respite from the drug causing HFS (13). In the event of a superficial disturbance of skin sensation, i.e., grade 1 HFS with no evident pain, a respite is presumably unnecessary. Patients are typically instructed by medical personnel to modify their routine, e.g., to avoid physical or thermal irritants, protect the skin, prevent a secondary infection, and avoid direct sunlight. The HFS Atlas (12) is a manual prepared by the manufacturer/distributor of capecitabine that broadly summarizes routine instructions
Table I. Baseline patient characteristics.

\begin{tabular}{|c|c|c|}
\hline Characteristics & Patients $(\mathrm{n}=90)$ & $\%$ \\
\hline \multicolumn{3}{|l|}{ Sex } \\
\hline Male & 54 & 60.0 \\
\hline Female & 36 & 40.0 \\
\hline \multicolumn{3}{|l|}{ Age (years) } \\
\hline Median & 66 & \\
\hline Range & $36-83$ & \\
\hline \multicolumn{3}{|l|}{ Body surface area $\left(\mathrm{m}^{2}\right)$} \\
\hline Median & 1.61 & \\
\hline Range & $1.16-2.09$ & \\
\hline \multicolumn{3}{|l|}{$\begin{array}{l}\text { Primary tumor site } \\
\text { and therapy regimen }\end{array}$} \\
\hline Colorectal & 79 & 87.8 \\
\hline capecitabine, oxaliplatin & 46 & \\
\hline capecitabine, oxaliplatin, bevacizumab & 25 & \\
\hline capecitabine, bevacizumab & 5 & \\
\hline capecitabine, irinotecan, bevacizumab & 3 & \\
\hline Stomach & 10 & 11.1 \\
\hline capecitabine, oxaliplatin & 6 & \\
\hline capecitabine, cisplatin, herceptin & 2 & \\
\hline capecitabine, herceptin & 1 & \\
\hline capecitabine, oxaliplatin, herceptin & 1 & \\
\hline Breast & 1 & 1.1 \\
\hline capecitabine & 1 & \\
\hline
\end{tabular}

Capecitabine cumulative dose ( $\mathrm{mg}$ )

Median

105,000

Range

$25,200-1,764,000$

Survey date (days)

Median

85

Range

$14-988$

Type of treatment

Adjuvant therapy

46

51.1

Palliative therapy

44

48.9

${ }^{a}$ Number of days after the commencement of capecitabine administration.

to prevent and treat HFS into 15 items. The current study examined the percentage of items in the HFS Atlas (12) that were (or were not) taken to prevent and treat HFS. Our results revealed that the percentage of items taken differed widely from 11.8 to $74.1 \%$.

The items that patients took most often were 'applying a moisturizer', 'keeping one's skin clean, e.g., washing one's hands and feet' and 'avoiding strenuous exercise (e.g., long walks, jogging, or aerobics)'. The items that patients took least often were 'using sunscreen on exposed areas' and 'using soft insoles'. The two major reasons why patients often applied a moisturizer were because multiple medical personnel clearly explained to patients that the moisturizer was prescribed and because patients were aware of how to specifically use the moisturizer. The rate at which patients took certain items was 
Table II. Association between the extent to which 15 items in the HFS Atlas were (or were not) taken and the development of symptoms of HFS.

\begin{tabular}{|c|c|c|c|c|c|c|}
\hline \multirow[b]{2}{*}{ Items } & \multicolumn{6}{|c|}{ HFS (pharmacist's assessment) } \\
\hline & $+(n=60)$ & $-(n=25)$ & Total $(\%)$ & Odds ratio & $95 \% \mathrm{CI}$ & P-value \\
\hline Applying a moisturizer & & & & 0.63 & $0.20-1.96$ & $0.424^{\mathrm{a}}$ \\
\hline+ & 43 & 20 & $63(74.1)$ & & & \\
\hline- & 17 & 5 & 22 & & & \\
\hline
\end{tabular}

Keeping one's skin clean,

$0.47 \quad 0.17-1.36-0.160^{\mathrm{a}}$

e.g., washing one's hands and feet

$+$

$-$

Avoiding strenuous exercise

(e.g., long walks, jogging or aerobics)

$+$

Avoiding activities that place pressure on the fingers or palms (e.g., acupressure and writing for long periods)

$+$

Wearing soft, comfortable footwear

$+$

$-$

Not wearing tight-fitting footwear or socks

$+$

Refraining from taking hot baths or showers

$+$

Avoiding wearing high-heeled shoes since they concentrate pressure on the toes

$+$

$-$

Being mindful of hot water when doing laundry by hand

$+$

$-$

Avoid direct sunlight (using an umbrella/hat/gloves/long sleeves)

$+$

Refraining from

wringing out a wet cloth by hand

$+$

14

46

Taking care to gently hold

a kitchen knife during use

$+$

$-$

Wearing thick cotton socks

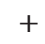

$+$

-

36

24

30

30

26

34

26

34

23

37

23

37

24

36

22

38

36
12

13

14
11

49

36 (42.4)

49

0.78

$0.27-2.26$

$0.649^{\mathrm{a}}$

21 (24.7)

64

0.48

$0.16-1.38$

$0.168^{a}$

19 (22.4)

66
8

17

4

21

19 (22.4)

$45 \quad 21 \quad 66$


Table II. Continued.

\begin{tabular}{|c|c|c|c|c|c|c|}
\hline \multirow[b]{2}{*}{ Items } & \multicolumn{6}{|c|}{ HFS (pharmacist's assessment) } \\
\hline & $+(n=60)$ & $-(n=25)$ & Total $(\%)$ & Odds ratio & $95 \% \mathrm{CI}$ & P-value \\
\hline Using sunscreen on exposed areas & & & & 0.81 & $0.22-2.97$ & $0.742^{\mathrm{c}}$ \\
\hline+ & 8 & 4 & $12(14.1)$ & & & \\
\hline- & 52 & 21 & 73 & & & \\
\hline Using soft insoles & & & & 0.36 & $0.10-1.39$ & $0.150^{\mathrm{c}}$ \\
\hline+ & 5 & 5 & $10(11.8)$ & & & \\
\hline - & 55 & 20 & 75 & & & \\
\hline
\end{tabular}

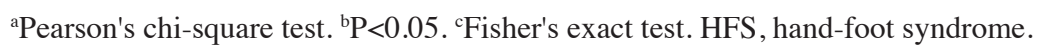

presumably affected by differences in how easy those items were for patients to take and in the manner in which medical personnel (which included pharmacists) gave instructions.

The relationship between the number of items taken to prevent and treat HFS and the development of symptoms of HFS was also examined. Our results indicated that patients who had developed symptoms of HFS had taken fewer items to prevent and treat HFS, while patients who did not develop symptoms of HFS had taken more items to prevent and treat HFS. In order to avoid the exacerbation of HFS, HFS must be prevented or the capecitabine dose must be reduced or halted in accordance with the symptoms. The current study covered a wide range of regimens, so the relationship between the development of HFS and a capecitabine respite or dose reduction was not examined. When examining a dose reduction or respite, a crucial aspect is whether numerous items have been taken to prevent HFS. The current study examined the relationship between the extent to which individual items in the HFS Atlas (12) were (or were not) taken and the development of symptoms of HFS.

Our results indicated that patients differed significantly in terms of three important items: 'avoiding activities that place pressure on the fingers or palms (e.g., acupressure and writing for long periods)' (the item taken fourth most often), 'not wearing tight-fitting footwear or socks' (the item taken sixth most often), and 'refraining from taking hot baths or showers' (the item taken seventh most often). Items that differed significantly included instructions to avoid external irritants such as physical and thermal irritants. Regarding other instructions, such as protecting the skin, preventing secondary infection, and avoiding direct sunlight, there were no significant differences in the development of HFS. Research has indicated that the hands are constantly exposed to external irritants and that symptoms are readily exacerbated in areas of the feet where pressure is concentrated or where shoes cause friction. These findings corroborate the importance of keeping the hands and feet still (14). The item that patients most often took to prevent and treat HFS was 'applying a moisturizer'. The application of an ointment containing heparin or an analogue, and urea cream or ointment, an ointment containing vitamin A, or petroleum jelly has been recommended, but the current results revealed no significant differences between whether these recommendations were followed and the development of HFS were observed.
Table III. Differences in a patient's subjective symptoms of HFS and a pharmacist's assessment of HFS.

\begin{tabular}{|c|c|c|c|c|c|}
\hline \multirow{2}{*}{$\begin{array}{l}\text { Patient's } \\
\text { subjective } \\
\text { symptoms }\end{array}$} & \multicolumn{2}{|c|}{ HFS } & \multirow[b]{2}{*}{ Odds ratio } & \multirow[b]{2}{*}{$95 \% \mathrm{CI}$} & \multirow[b]{2}{*}{ P-value } \\
\hline & $\begin{array}{c}+ \\
(n=63)\end{array}$ & $\begin{array}{c}- \\
(n=27)\end{array}$ & & & \\
\hline & & & 11.76 & $2.91-47.62$ & $0.0002^{\mathrm{a}}$ \\
\hline+ & 60 & 17 & & & \\
\hline- & 3 & 10 & & & \\
\hline
\end{tabular}

Fisher's exact test was performed. ${ }^{\mathrm{P}}<0.001$. HFS, hand-foot syndrome.

The differences in a patient's subjective symptoms of HFS and a pharmacist's assessment of HFS were also examined. Our results indicated that $20 \%$ of the patients reported symptoms of HFS even though a pharmacist found no such symptoms. Based on this finding, patients may notice symptoms of HFS earlier than the assessment with CTCAE Version 4.0 by medical personnel (including pharmacists) indicates. This reveals the need for improved assessment criteria as well as a more concerted effort to obtain detailed information from patients.

The current study has several limitations. First, this study was a cross-sectional survey; therefore, the cumulative dose of capecitabine was not uniform at the time of the study. This calls into question the reliability of the cumulative dose of capecitabine at which symptoms of HFS developed or did not develop. HFS due to capecitabine is considered to result from a cumulative toxicity. One study reported that a median cumulative dose of capecitabine of $56,000 \mathrm{mg} / \mathrm{m}^{2}$ results in an incidence of symptoms of grade 1 HFS of $80 \%$ or higher, while another study reported that a median cumulative dose of capecitabine of 100,000 or $200,000 \mathrm{mg} / \mathrm{m}^{2}$ results in an incidence of symptoms of grade 1 HFS of $90 \%$ or higher (15). When a study is conducted early on in treatment, assessing whether items to treat and prevent HFS are effective or not is difficult, as is determining whether symptoms of HFS have yet to develop. This is true regardless of whether or not items to treat and prevent HFS are actually effective. Second, the risk of 
developing HFS will presumably differ as a result of differences in patient characteristics, and the current analysis did not take that into account. Studies have reported that the elderly, patients with renal dysfunction, and patients with anemia have a high incidence of grade 2 or more severe HFS (15). Studies have also reported that diabetes mellitus, concomitant administration of bevacizumab and capecitabine, history of receiving a fluoropyrimidine drug, and early onset of symptoms (within 21 days) are potential risk factors for the development of HFS (14). Third, the number of patients analyzed was as small as 90 patients. Our sample size was the number of patients recruited at 4 facilities (key hospitals for cancer care). The sample size needs to be increased, and the current findings need to be verified in future studies. In light of these points, the current finding of a relationship between the extent to which items to prevent and treat HFS were (or were not) taken and the development of symptoms of HFS is not generalizable. However, the current study is significant in the sense that a cross-sectional survey conducted at multiple facilities revealed a relationship between the rates at which patient education to prevent HFS was implemented in routine practice and the development of HFS. The novelty of this study is not only from the drugs but also from other 15 aspects including patient behaviors, which suggests the importance of the management is patient's behavior which patients tend to ignore.

In the present study, medical personnel specifically checked which items were taken by patients. Based on our findings, instructions emphasizing that patients take all necessary items to prevent and treat HFS and that they avoid external irritants, which are highly likely to influence the development of symptoms of HFS, lead to a qualitative improvement in the management of HFS. Therefore, our findings are highly applicable in clinical settings.

\section{Acknowledgements}

Not applicable.

\section{Funding}

No funding was received.

\section{Availability of data and material}

The datasets during and/or analyzed during the current study available from the corresponding author on reasonable request.

\section{Authors' contributions}

SS contributed to data collection and interpretation, and wrote the initial draft of the manuscript. SN, YI, DS, JK and DI contributed to data collection and interpretation, and critically reviewed the manuscript. KT and KI contributed to analysis and interpretation of data, and assisted in the preparation of the manuscript. FS and HS contributed to management of data, and critically reviewed the manuscript. TY designed the study, and contributed to analysis and interpretation of data, and assisted in the preparation of the manuscript. All authors prepared the survey sheet. All authors read and approved the final manuscript.

\section{Ethics approval and consent to participate}

This study was ethically reviewed and approved by the Ethics Committee of Keiyu Hospital, Showa University Northern Yokohama Hospital, Yokohama Rosai Hospital, Fujisawa City Hospital, and Hoshi University. Written informed consent was obtained from all patients.

\section{Patient consent for publication}

Not applicable.

\section{Competing interests}

The authors declare that they have no competing interests.

\section{References}

1. Twelves C, Wong A, Nowacki MP, Abt M, Burris H 3rd, Carrato A, Cassidy J, Cervantes A, Fagerberg J, Georgoulias V, et al: Capecitabine as adjuvant treatment for stage III colon cancer. N Engl J Med 352: 2696-2704, 2005.

2. Watanabe T, Itabashi M, Shimada Y, Tanaka S, Ito Y, Ajioka Y, Hamaguchi T, Hyodo I, Igarashi M, Ishida H, et al: Japanese Society for Cancer of the Colon and Rectum (JSCCR) Guidelines 2014 for treatment of colorectal cancer. Int J Clin Oncol 20: 207-239, 2015.

3. Tsuboi H, Yonemoto K and Katsuoka K: A case of bleomycin-induced acral erythema (AE) with eccrine squamous syringometaplasia (ESS) and summary of reports of AE with ESS in the literature. J Dermatol 32: 921-925, 2005.

4. Nikolaou V, Syrigos K and Saif MW: Incidence and implications of chemotherapy related hand-foot syndrome. Expert Opin Drug Saf 15: 1625-1633, 2016

5. Mortimer JE, Lauman MK, Tan B, Dempsey CL, Shillington AC and Hutchins KS: Pyridoxine treatment and prevention of handand-foot syndrome in patients receiving capecitabine. J Oncol Pharm Pract 9: 161-166, 2003.

6. Lassere $Y$ and Hoff $P$ Management of hand-foot syndrome in patients treated with capecitabine (Xeloda). Eur J Oncol Nurs 8: S31-S40, 2004.

7. Gressett SM, Stanford BL and Hardwicke F: Management of hand-foot syndrome induced by capecitabine. J Oncol Pharm Pract 12: 131-141, 2006.

8. Nagore E, Insa A and Sanmartín O: Antineoplastic therapy-induced palmar plantar erythrodysesthesia ('hand-foot') syndrome. Incidence, recognition and management. Am J Clin Dermatol 1: 225-234 (2000).

9. Nakane M: Neurotoxicity and Dermatologic Toxicity of Cancer Chemotherapy. Jpn J Cancer Chemother 33: 29-33, 2006.

10. Mae Y, Yokokawa T, Kawakami K, Yagi N, Suenaga M, Shinozaki E, Matsusaka S, Mizunuma N and Hama T: Usefulness of Pharmaceutical Outpatient Clinic in XELOX Therapy. Jpn J Pharm Health Care Sci 37: 611-615, 2011.

11. Morimoto E and Inoue N: Current state of nursing care for prevention of hand-foot-syndrome from capecitabine, from the study for certified nurse in cancer chemotherapy nursing in Japan. J Jpn Soc Cancer Nurs 28: 30-36, 2014.

12. Chugai Pharmaceutical Co. Ltd: Hand-Foot Syndrome Atlas. 3rd edition, Tokyo, 2011.

13. Yamazaki $\mathrm{N}$ and Taguchi T: Hand-foot syndrome. J Clin Exp Med 216: 257-260, 2006.

14. Yokokawa T, Kawakami K, Mae Y, Sugita K, Watanabe H, Suzuki K, Suenaga M, Mizunuma N, Yamaguchi T and Hama T: Risk factors exacerbating hand-foot skin reaction induced by capecitabine plus oxaliplatin with or without bevacizumab therapy, Ann Pharmacother 49: 1120-1124, 2015.

15. Scheithauer W, McKendrick J, Begbie S, Borner M, Burns WI, Burris HA, Cassidy J, Jodrell D, Koralewski P, Levine EL, et al: Oral capecitabine as an alternative to i.v. 5-fluorouracil-based adjuvant therapy for colon cancer: safety results of a randomized, phase III trial. Ann Oncol 14: 1735-1743, 2003. 